\title{
Fallopian Tube Tumor Mimicking Primary Gastrointestinal Malignancy
}

\author{
Anupam K. Gupta ${ }^{1}$, Oscar A. Vazquez ${ }^{2}$ \\ 1. Minimally Invasive Surgery, University of Miami Hospital, Miami, USA 2. Surgery, Charles E. Schmidt College of \\ Medicine, Florida Atlantic University, Boca Raton, USA
}

Corresponding author: Anupam K. Gupta, dranupamkumargupta@gmail.com

\begin{abstract}
A 67-year-old female patient positive for a mismatch repair gene mutation and history of serous carcinoma of the fallopian tube presented with a lower gastrointestinal bleed. Clinical workup was suggestive of a primary gastrointestinal malignancy. Pathological review after right hemicolectomy revealed the primary tumor was a fallopian tube carcinoma. Over the next few years, she presented with upper and lower gastrointestinal bleeds from a recurrent metastatic disease, which was from the primary fallopian tube cancer. Although serous carcinoma of the fallopian tube is not an uncommon diagnosis, it is unusual for it to present with symptoms of recurrent gastrointestinal bleed mimicking a primary gastrointestinal malignancy.
\end{abstract}

Categories: Obstetrics/Gynecology, General Surgery, Oncology

Keywords: malignancy, gi bleed, fallopian tube, tumor, carcinoma

\section{Introduction}

Primary fallopian tube carcinoma (PFTC) is a sporadic gynecologic malignant tumor and accounts for approximately $0.14 \%-1.8 \%$ of female genital malignancies [1-3]. The most common route of dissemination is metastasis through the peritoneal cavity with rare instances of hematogenous spread. Distant metastasis often occurs in the liver, brain, or lung [4-6]. Gynecological malignancies in their advanced stages are known to cause bowel obstruction and, most commonly it will cause bowel involvement with extrinsic compression, adhesion, or carcinomatosis [7]. It is unusual for gynecological malignancy to present with gastrointestinal (GI) bleeding mimicking primary colon cancer as it has been reported very few times in the literature, with one case of metastatic ovarian carcinoma presenting as sigmoid colon malignancy [6]. It is also uncommon for it to present with invasion into the bowel wall with a recurrent GI bleed.

\section{Case Presentation}

A 67-year-old female patient with a history of glaucoma and left fallopian tube serous adenocarcinoma status post total abdominal hysterectomy and right salpingo-oophorectomy presented with symptoms of lower GI bleeding and anemia for which she underwent colonoscopy. Her family history was positive for endometrial cancer in her sister, and genetic studies showed mutations in mismatch repair genes suggestive of hereditary non-polyposis colorectal cancer (Lynch syndrome - MLH1/MSH2/MSH6/PMS2). Immunohistochemistry of the fallopian tube tumor was positive for Ber-Ep4, EMA, and WT1. It was negative for calretinin with some cells positive for estrogen receptor (ER) immunostain (consistent with high-grade distributed under the terms of the Creative Commons Attribution License CC-BY 4.0., which permits unrestricted use, distribution, and reproduction in any medium, provided the original author and source are credited. serous adenocarcinoma of Müllerian origin). Colonoscopy revealed that the patient had a proximal transverse colon mass, which appeared as a primary colon malignant mass (Figure 1). Assuming this was primary colon malignancy, she underwent right hemicolectomy with the intraoperative presence of enlarged lymphatics along the ileocolic trunk. She had elevated CA-125 levels, and carcinoembryonic antigen levels were within normal limits. Pathology showed poorly differentiated carcinoma involving right pericolic adipose tissue (Figure 2) suggestive of primary fallopian cancer origin. The patient was discharged in stable condition and placed on gemcitabine chemotherapy after this for which she completed three out of six cycles. 


\section{Cureus}

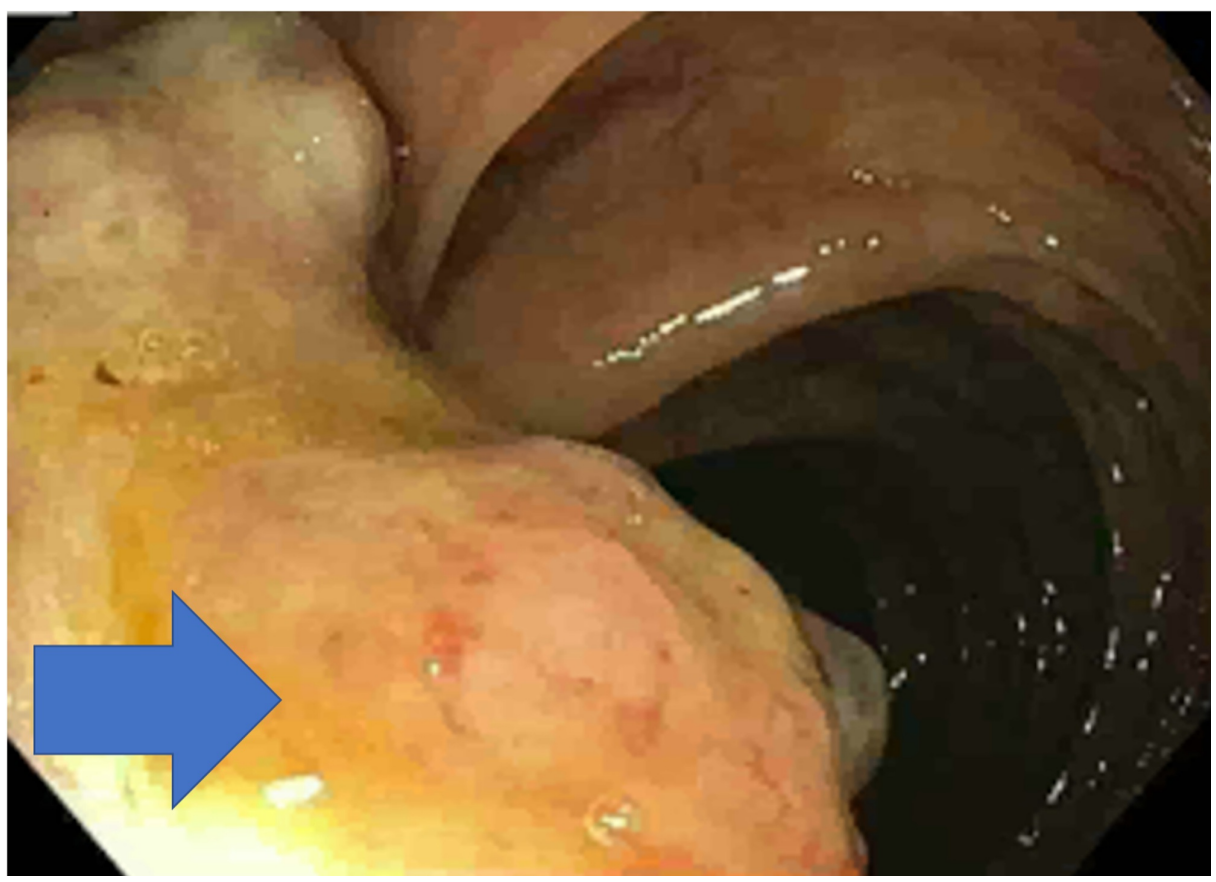

FIGURE 1: Colonosopy showing ulcerated mass in transverse colon (blue arrow).

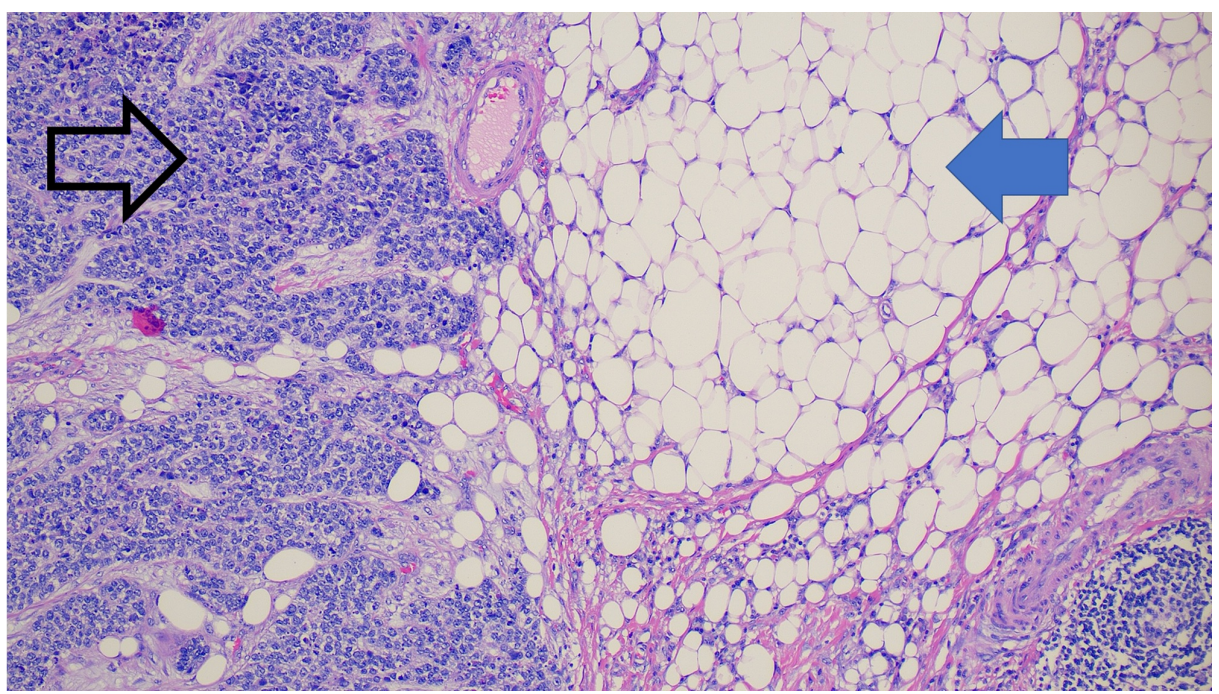

FIGURE 2: Hematoxilyn and eosin stain showing poorly differentiated carcinoma (black arrow) involving right pericolic adipose tissue (blue arrow).

Two years from the right hemicolectomy, the patient presented with melena, weakness, hypotension, fatigue, and a hemoglobin of $5.3 \mathrm{~g} / \mathrm{dL}$. An upper GI endoscopy revealed an ulcerated mass in the second part of the duodenum (Figure 3). Biopsy of the duodenal growth showed poorly differentiated carcinoma (Figure 4). Two months later, the patient presented with cachexia and a lower GI bleed where a colonoscopy revealed a rectal growth with pathology report confirming fallopian tube origin (Figure 5). Ultimately, the patient denied colonoscopy, or any surgical intervention, and she was discharged to a hospice center where she subsequently succumbed to extensive metastatic tumor burden. 


\section{Cureus}

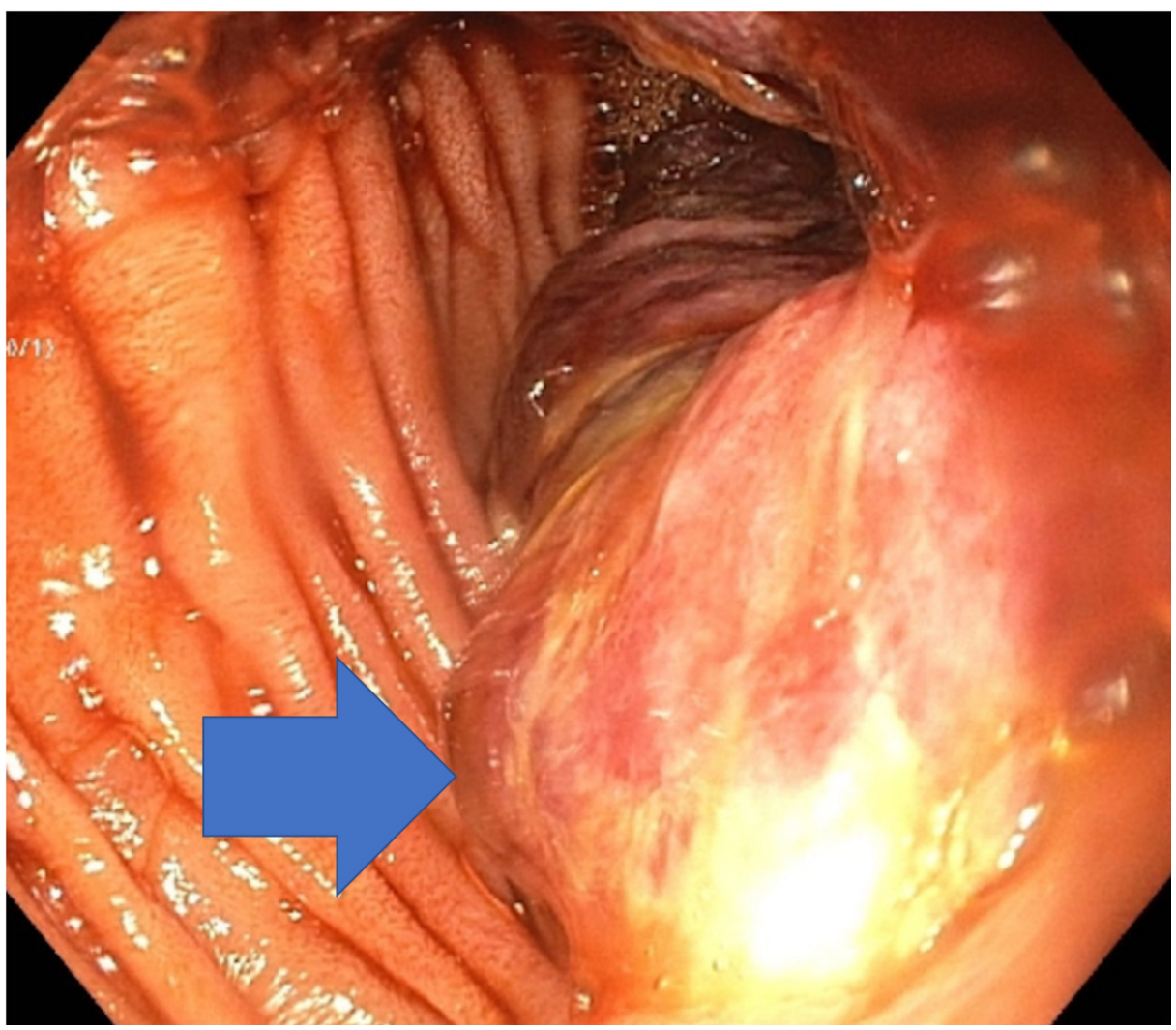

FIGURE 3: Colonoscopy showing mass at the second part of the duodenum (blue arrow).

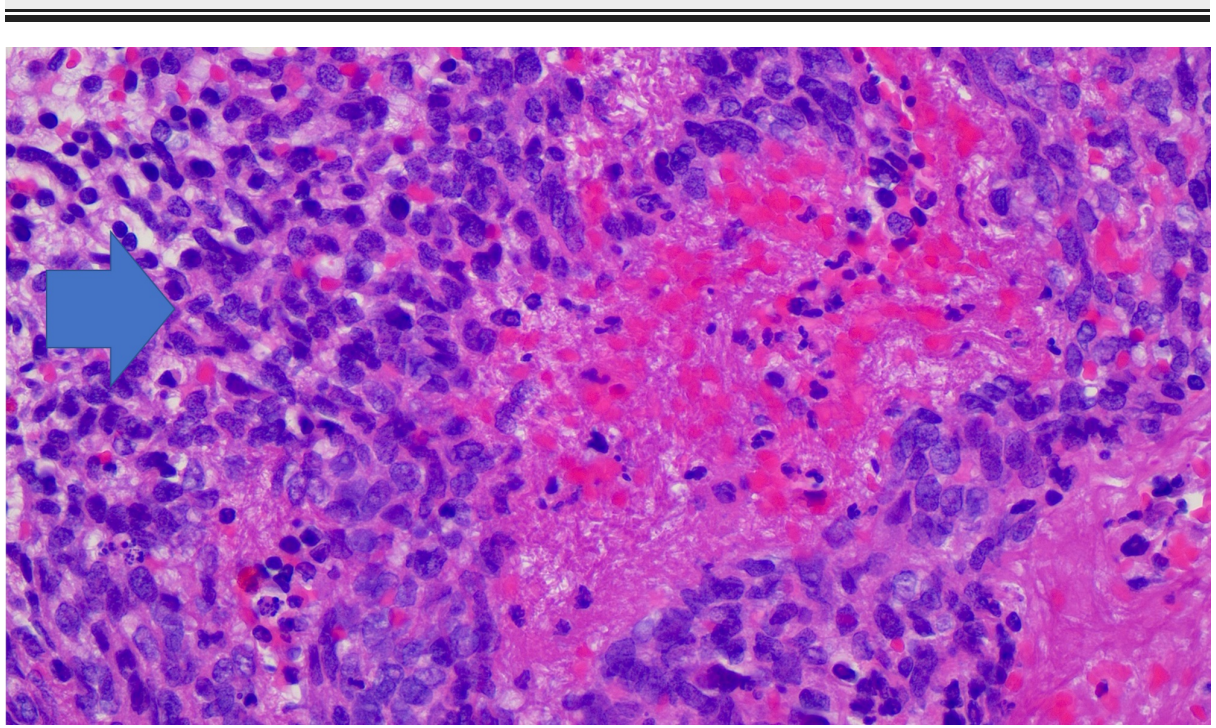

FIGURE 4: Hematoxylin and eosin stain showing poorly differentiated carcinoma (blue arrow). 


\section{Cureus}

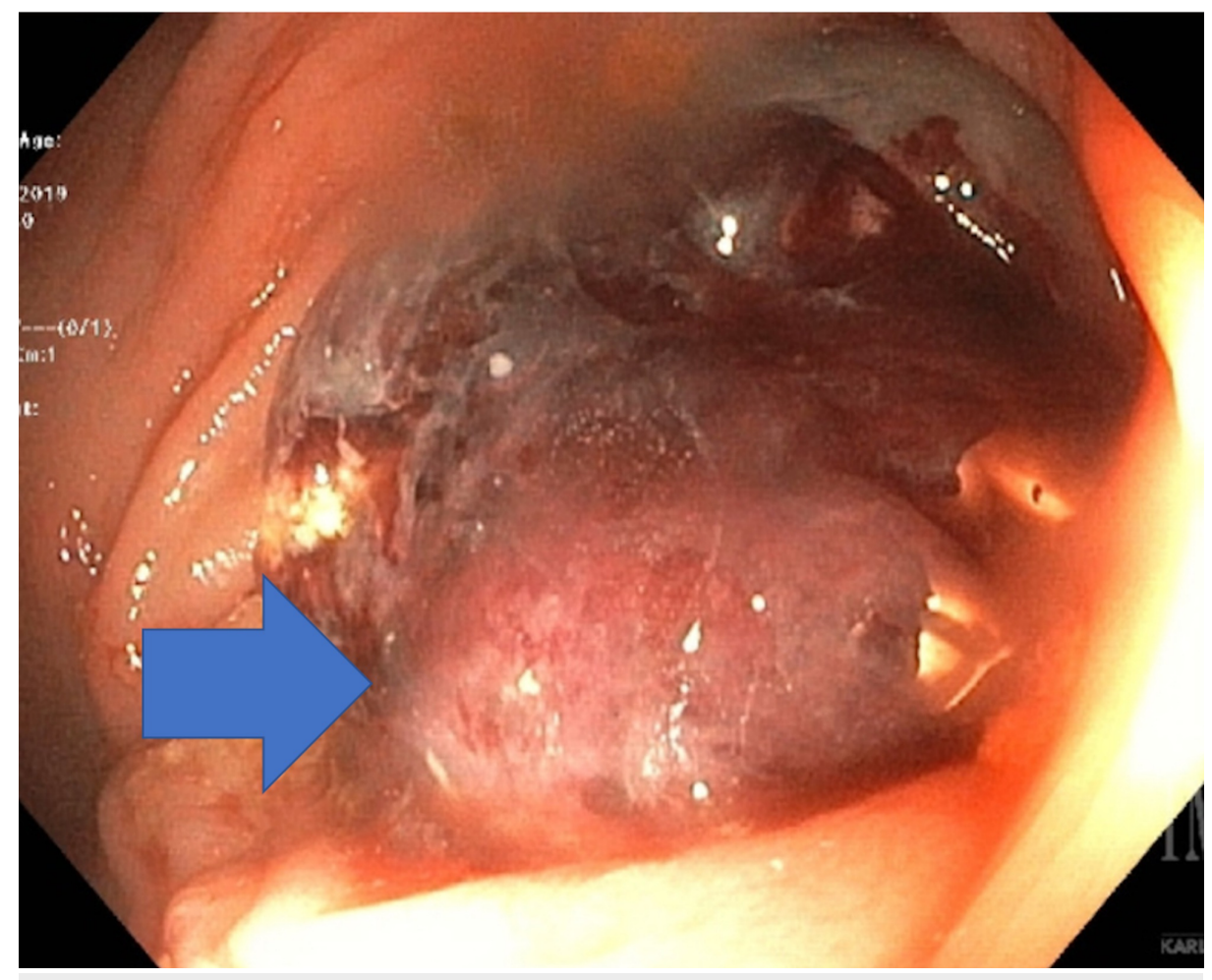

FIGURE 5: Colonoscopy showing rectal growth of fallopian tube origin (blue arrow).

\section{Discussion}

PFTC histologically and clinically resembles ovarian epithelial carcinoma and presents within the peritoneum where it stays throughout its course. It can metastasize to other peritoneal surfaces by exfoliating cells that implant throughout the cavity with the intraperitoneal route of dissemination being considered as the most common [8-10]. Its incidence has been rising and varies between 2.9/1,000,000 and $5.7 / 1,000,000$ during the last decades [11]. Up to $40 \%-60 \%$ of high-grade serous carcinomas of the ovary or peritoneum may have fallopian tube fimbriae origin as per genetic, molecular, and histologic evidence [12]. Chronic tubal inflammation, infertility, tuberculous salpingitis, and tubal endometriosis can be associated with PFTC, but the exact etiology is still unknown, though it is associated with BRCA germline and TP53 mutations (similarly to ovarian carcinoma) [13-15].

Alhough PFTC can metastasize to the colon typically, the GI involvement is usually limited to the seromuscular layer of the small and large bowel and its mesentery [16]. However, in our patient, the presentation in the right colon mimicking a primary and histopathology is suggestive of the possible lymphatic route or spread, which is unusual. Kadakia et al. described the most common endoscopic findings of metastatic GI malignancies as ulcerated nodules, ulcerated submucosal masses, umbilicated nodules with central exudate, and necrotic ulcers with heaped-up margins [17]. This morphology is consistent with the duodenal and rectal metastasis found on upper and lower endoscopy later in this patient's course. As mentioned by Park et al., it is essential to identify the primary malignancy early, especially in a patient like ours, with a history of mismatch repair gene mutation and PFTC diagnosis [6]. This is important because it can help guide treatment options since colorectal cancer is usually treated with 5 -fluorouracil and platinum agents, as opposed to fallopian tube cancer which is treated with paclitaxel and platinum agents $[12,18]$

\section{Conclusions}

PFTC is a rare tumor that can metastasize by an intraperitoneal route. It is unusual for it to present with recurrent GI bleeding mimicking a primary GI malignancy. Management is guided by identifying tumor origin based on history of other primary malignancies and they should be ruled out, even in a single intraluminal lesion.

\section{Additional Information}

\section{Disclosures}

Human subjects: Consent was obtained by all participants in this study. Conflicts of interest: In compliance with the ICMJE uniform disclosure form, all authors declare the following: Payment/services 
info: All authors have declared that no financial support was received from any organization for the submitted work. Financial relationships: All authors have declared that they have no financial relationships at present or within the previous three years with any organizations that might have an interest in the submitted work. Other relationships: All authors have declared that there are no other relationships or activities that could appear to have influenced the submitted work.

\section{References}

1. Kalampokas E, Kalampokas T, Tourountous I: Primary fallopian tube carcinoma . Eur J Obstet Gynecol Reprod Biol. 2013, 169:155-161. 10.1016/j.ejogrb.2013.03.023

2. Lau HY, Chen YJ, Yen MS, Chen RF, Yeh SO, Twu NF: Primary fallopian tube carcinoma: a clinicopathologic analysis and literature review. J Chin Med Assoc. 2013, 76:583-587. 10.1016/j.jcma.2013.06.010

3. Ma Y, Duan W: Clinical and survival analysis of 36 cases of primary fallopian tube carcinoma . World J Surg Oncol. 2014, 12:311. 10.1186/1477-7819-12-311

4. Tan DS, Agarwal R, Kaye SB: Mechanisms of transcoelomic metastasis in ovarian cancer . Lancet Oncol. 2006, 7:925-934. 10.1016/S1470-2045(06)70939-1

5. Lengyel E: Ovarian cancer development and metastasis. Am J Pathol. 2010, 177:1053-1064. 10.2353/ajpath.2010.100105

6. Park JH, Jung DH, Baek JH: Metastatic colon cancer of an ovarian cancer origin mimicking primary colon cancer: a case report. Korean J Clin Oncol. 2018, 14:53-57. 10.14216/kjco.18009

7. Lee YC, Jivraj N, O'Brien C, et al.: Malignant bowel obstruction in advanced gynecologic cancers: an updated review from a multidisciplinary perspective. Obstet Gynecol Int. 2018, 2018:1867238. 10.1155/2018/1867238

8. Rexhepi M, Trajkovska E, Ismaili H, Besimi F, Rufati N: Primary fallopian tube carcinoma: a case report and literature review. Open Access Maced J Med Sci. 2017, 5:344-348.

9. Dauplat J, Hacker NF, Nieberg RK, Berek JS, Rose TP, Sagae S: Distant metastases in epithelial ovarian carcinoma. Cancer. 1987, 60:1561-1566. 10.1002/1097-0142(19871001)60:7<1561::AIDCNCR2820600725>3.0.CO;2-V

10. Cormio G, Rossi C, Cazzolla A, Resta L, Loverro G, Greco P, Selvaggi L: Distant metastases in ovarian carcinoma. Int J Gynecol Cancer. 2003, 13:125-129. 10.1136/ijgc-00009577-200303000-00004

11. Riska A, Leminen A: Determinants of incidence of primary fallopian tube carcinoma (PFTC) . Methods Mol Biol. 2009, 472:387-396.

12. Berek JS, Crum C, Friedlander M: Cancer of the ovary, fallopian tube, and peritoneum . Int J Gynaecol Obstet. 2015, 131:S111-S122. 10.1016/j.ijgo.2015.06.007

13. Mladenovic-Segedi L: Primary fallopian tube carcinoma . Med Pregled. 2009, 62:31-36. 10.2298/MPNS0902031M

14. Howitt BE, Hanamornroongruang S, Lin DI, et al.: Evidence for a dualistic model of high-grade serous carcinoma: BRCA mutation status, histology, and tubal intraepithelial carcinoma. Am J Surg Pathol. 2015, 39:287-293. 10.1097/PAS.0000000000000369

15. Quartuccio SM, Karthikeyan S, Eddie SL, et al.: Mutant p53 expression in fallopian tube epithelium drives cell migration. Int J Cancer. 2015, 137:1528-1538. 10.1002/ijc.29528

16. Taylor RR, Phillips WS, O'Connor DM, Harrison CR: Unusual intramural gastric metastasis of recurrent epithelial ovarian carcinoma. Gynecol Oncol. 1994, 55:152-155. 10.1006/gyno.1994.1266

17. Kadakia SC, Parker A, Canales L: Metastatic tumors to the upper gastrointestinal tract: endoscopic experience. Am J Gastroenterol. 1992, 87:1418-1423.

18. Haydon A: Adjuvant chemotherapy in colon cancer: what is the evidence? . Intern Med J. 2003, 33:119-124. 10.1046/j.1445-5994.2003.00324.x 